Consequently, either the hypothesis of linear dependency has led to a contradiction so that the three columns are linearly independent, or alternatively Eq. (6) must hold. But if Eq. (6) holds, then

$$
c_{i} x_{i}^{\prime \prime}=c_{i} x_{i}^{\prime} \quad \text { for all } A,
$$

and $A$ can be chosen so that at least one of $x_{2}^{\prime \prime}, x_{3}^{\prime \prime}, x_{8}^{\prime \prime}$ vanishes, so that an equivalent solution involving fewer columns has been obtained; the same analysis may now be applied to the new solution.

It follows that, eventually, an equally good solution utilieing only linearly independent columns will be obtained. Since not more than $m$ columns can be linearly independent, the theorem is proved.

\title{
REFeRENCES
}

1. G. B. Dantzig, Maximization of a linear form whose variables are subject to a system of linear inequalities, Headquarters, U.S.A.F., 1949

2. A. Charnes, W. W. Cooper, A. Henderson, An introduction to linear programming, 1953, Wiley, N.Y.

\section{HEAT FLOW IN A HALF SPACE*}

By WALTER P. REID† (U. S. Naval Ordnance Test Station, China Lake, California)

This heat flow problem has a more difficult boundary condition than usual. A formal solution is obtained by the combined use of Laplace and Fourier sine transforms.

Let the region $x>0$ have an initial temperature which is a function of $x$ only, $f(x)$. Assume that there is radiation of heat from the surface $x=0$ to a finite slab, and from the other surface of the slab to surroundings whose temperature is a prescribed function of the time, $g(t)$. The slab is assumed to be thin and of high thermal conductivity so that its temperature may be considered to be uniform throughout at any time. The heat exchange by radiation between adjacent surfaces will be taken to be proportional to the difference in temperatures of the two surfaces. Mathematically, then, the problem may be stated as follows:

$$
\begin{gathered}
\frac{\partial u(x, t)}{\partial t}=\kappa \frac{\partial^{2} u(x, t)}{\partial x^{2}} \quad \text { for } \quad x>0, t>0, \\
u(x, 0)=f(x), \\
\frac{\partial u(0, t)}{\partial x}=a[u(0, t)-v(t)], \quad t>0 \\
h \frac{\partial v(t)}{\partial t}=a[u(0, t)-v(t)]-b[v(t)-g(t)], \quad t>0 \\
v(0)=V .
\end{gathered}
$$

${ }^{*}$ Received June 13, 1955.

†Now at Los Alamos Scientific Laboratory, Los Alamos, New Mexico. 
In these equations $u(x, t)$ is the temperature at some point in the region $x>0$, while $v(t)$ is the temperature of the slab. The quantities $a, b, h$ and $\kappa$ are assumed to be positive constants.

In the following both the Laplace transform and the Fourier sine transform will be used. This brings up the question of notation. A bar above the letter is sometimes used to represent a transform, or a change from capital letter to lower case, or a change in letter altogether. These methods are all satisfactory if just a single transform is used, but have disadvantages in the present case. In Eq. (16), for example, notation for three different transforms is needed. In this paper a transform will be indicated simply by changing the letter used in the argument of the function, as follows:

$$
\begin{gathered}
u(x, s) \equiv \int_{0}^{\infty} e^{-s t} u(x, t) d t, \\
u(\xi, t) \equiv \int_{0}^{\infty} u(x, t) \sin \xi x d x .
\end{gathered}
$$

The Laplace transforms of Eqs. (1), (3) and (4) are:

$$
\begin{gathered}
s u(x, s)-f(x)=\kappa \frac{\partial^{2} u(x, s)}{\partial x^{2}}, \\
\frac{\partial u(0, s)}{\partial x}=a[u(0, s)-v(s)], \\
h s v(s)-h V=a[u(0, s)-v(s)]-b[v(s)-g(s)] .
\end{gathered}
$$

Hence

$$
\begin{aligned}
{\left[a b-(a+b) \partial / \partial x+a h \kappa \partial^{2} / \partial x^{2}-h \kappa \partial^{3} / \partial x^{3}\right] u(0, s) } & \\
& =a b g(s)-a h f(0)+h f^{\prime}(0)+a h V .
\end{aligned}
$$

Let

$$
w(x, t)=\left[a b-(a+b) \partial / \partial x+a h \kappa \partial^{2} / \partial x^{2}-h_{\kappa} \partial^{3} / \partial x^{3}\right] u(x, t) .
$$

Then

$$
\begin{gathered}
\frac{\partial w(x, t)}{\partial t}=\kappa \frac{\partial^{2} w(x, t)}{\partial x^{2}}, \\
s w(x, s)-w(x, 0)=\kappa \frac{\partial^{2} w(x, s)}{\partial x^{2}}, \\
s w(\xi, s)-w(\xi, 0)=\kappa \xi w(0, s)-\xi^{2} \kappa w(\xi, s), \\
w(\xi, s)=\frac{w(\xi, 0)+\kappa \xi w(0, s)}{s+\kappa \xi^{2}} .
\end{gathered}
$$

From Eq. (11):

$$
w(0, s)=a b g(s)-a h f(0)+h f^{\prime}(0)+a h V .
$$


Take the Fourier sine transform of Eq. (12) and then eliminate the derivatives of $u$ by integrating by parts. This gives

$$
\begin{aligned}
w(\xi, t)=\int_{0}^{\infty} u(x, t)\left[a\left(b-h \kappa \xi^{2}\right) \sin \xi x+\xi(a+b\right. & \left.\left.-h_{\kappa} \xi^{2}\right) \cos \xi x\right] d x \\
& +a h_{\kappa} \xi u(0, t)-h_{\kappa \xi} \frac{\partial u(0, t)}{\partial x} .
\end{aligned}
$$

Let

$$
\phi(\xi)=\int_{0}^{\infty} f(x)\left[a\left(b-h \kappa \xi^{2}\right) \sin \xi x+\xi\left(a+b-h \kappa \xi^{2}\right) \cos \xi x\right] d x
$$

Then

$$
w(\xi, 0)=\phi(\xi)+a h \kappa \xi f(0)-h \kappa \xi f^{\prime}(0)
$$

So

$$
w(\xi, s)=\frac{\phi(\xi)+\kappa \xi a b g(s)+\kappa \xi a h V}{s+\kappa \xi^{2}}
$$

Hence

$$
w(\xi, t)=[\phi(\xi)+\kappa \xi a h V] \exp \left(-\kappa \xi^{2} t\right)+\kappa \xi a b \int_{0}^{t} g(\tau) \exp \left[-\kappa \xi^{2}(t-\tau)\right] d \tau .
$$

But

$$
w(x, t)=\frac{2}{\pi} \int_{0}^{\infty} w(\xi, t) \sin \xi x d \xi
$$

Therefore, from Eqs. (12) and (23),

$$
u(x, t)=\frac{2}{\pi} \int_{0}^{\infty} w(\xi, t) \frac{a\left(b-h_{\kappa} \xi^{2}\right) \sin \xi x+\xi\left(a+b-h_{\kappa} \xi^{2}\right) \cos \xi x}{a^{2}\left(b-h_{\kappa} \xi^{2}\right)^{2}+\xi^{2}\left(a+b-h_{\kappa} \xi^{2}\right)^{2}} d \xi,
$$

where $w(\xi, t)$ is given by Eq. (22), and $\phi(\xi)$ by Eq. (19). From Eq. (3) and (24) one may obtain $v(t)$.

\section{NOTE ON THE SYMMETRICAL PROPERTY OF THE THERMAL CONDUCTIVITY TENSOR*}

\section{By M. LESSEN (University of Pennsylvania)}

According to the Onsager reciprocal hypothesis, the thermal conductivity tensor among other similar properties of matter is symmetrical. In the following note it is demonstrated that for some cases of interest, the property of symmetry according to the Onsager hypothesis is not a necessary piece of information.

Assuming a space filled with a continuous medium at temperature $T$, having a

*Received June 15, 1955. 